\title{
On the Use of Modal Powers and Energies in the Analysis and Design of Metamaterial Structures
}

\author{
M. Hassanein RABAH, Divitha SEETHARAMDOO and Chukwuka OZUEM \\ LEOST-COSYS, IFSTTAR, Villeneuve d'Ascq, France \\ divitha.seetharamdoo@ifsttar.fr
}

\begin{abstract}
This paper discusses the problem of integrating metamaterial (MTM) structures with antennas. We present a new approach based on the computation of the energies of the surface current modes by means of the theory of characteristic modes (TCM). We also introduce new simple formulation to compute the stored energy using the impedance operation of the method of moment (MoM). This analysis can be done the antenna (driven element) and the inclusion (resonant element).
\end{abstract}

Index Terms-ESA, stored energy, metamaterial-inspiredantennas, characteristic modes, method of moments

\section{INTRODUCTION}

Nowadays, an antenna occupies up to $20 \%$ of the overall size of a mobile terminal. Therefore, their a real need to miniaturise antenna size, since the antenna allocated area imposes a significant limitation on the overall size of a portable wireless system. MTM inspired concept has been proposed to miniaturize antennas suffering from their small electrical sizes while keeping a good radiation efficiency [1]. The idea of MTM inspired antennas is to associate a driven element to a resonant parasitic element in the very near field of the driven element. These electrically small antennas (ESAs) are nearly completely matched to a real source and have a very high overall efficiency. These properties are achieved through the parasitic element, which replaces the need for an external matching network and which works with the driven element to enhance the radiation process. Fig. 1 illustrates the coupling and radiation behavior of a small-loaded antenna. In

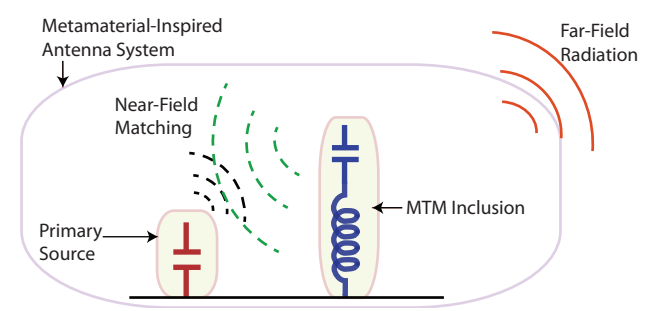

Fig. 1. Illustration of the coupling and radiation behavior of the nearfield metamaterial-inspired small antenna. The driven element (capacitor) represents a monopole/dipole antenna which stores electric energy in his near-field. The parasitic element, replaces the need for an external matching network.

fact, electrically small antennas suffer from the high reactive energy in their near field. This energy could be electric, then modelled by a capacitor (Fig. 1) or magnetic and modelled by an inductor. However, each case needs a specific parasitic element in the near field to behave like and MNG and ENG medium, respectively. On the other hand, antenna systems become progressively smaller and arbitrary shaped when they are integrated into the chassis of the terminal. Hence, Erentok's technique cannot be used to enhance the efficiency. More precisely, it is not easy to identify whether the inclusion must be capacitive or inductive at a specific frequency. This problem is illustrated in Fig. 2.

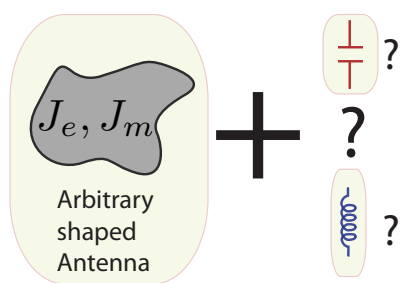

Fig. 2. Illustration of the problem of associating metamaterial inclusion to non-classical arbitrary shaped antennas.

A series of our works aim to propose a systematic methodology to generalise the MTM-inspired antennas concept to any possible type of antennas [2], [3], [4], [5], [6], [7]. The main idea consists at a first level of a separated energetic study of the antenna (driven element) and the inclusion (parasitic element), then a complete modal study of the overall antenna system. In general the driven element is electrically small, thus it operates as a mono-mode antenna. The type of the energy stored by this mode will determine which type of inclusion need to be used. For instance, if the total reactive power is electrical, thus an inclusion with dominant magnetic stored energy must be associated and vice-versa .

In this context, this paper presents a simplified formulation of the modal energies using the impedance matrix computed using the method of moment (MoM). Further studies of MTM inspired structures such as S-shaped inclusion and meander line will be presented during the talk.

\section{Formulation of Modal EnERgies BASED ON THE IMPEDANCE MATRIX}

The theory of characteristic mode was given for perfectly conducting (PEC) objects [8], and for penetrable objects [9]. Any applied electrical field $\underline{E}^{\text {inc }}$ will produce a surface current $I$ over a conducting surface $S$. From Maxwell equations and the boundary conditions derived therein, the total tangential electric field must be zero on the surface of the conductor. 
Thus, this surface currents on the conductor must produce a scattered field that enforces this boundary condition. The surface currents generated in this way are defined using the operator $Z$ with impedance dimensions: $\left[Z(\underline{I})-\underline{E}^{\text {inc }}\right]_{\tan }=0$. Since the modal currents are weighted and orthogonal over the surface $S$, any arbitrary current can be expanded as follows:

$$
\underline{I}=\sum_{n=1}^{\infty} \alpha_{n} \underline{I}_{n} \quad \mathrm{n}=1,2,3, \ldots
$$

$\alpha_{n}$ are the weighting factors. These modal currents are the eigenmodes of the structure and they are calculated by solving the generalised eigenvalue problem. More details on TCM can be found in [10].

When implemented using MoM, the impedance operator become the impedance matrix [Z] such as:

$$
[X][I]=\lambda[R][I]
$$

Where $\mathrm{X}$ and $\mathrm{R}$ are the imaginary and real part of the impedance matrix respectively, and $I$ are the eigenvalue and eigen-current (used in the stored energy calculation) respectively.

In [11], [12] the expression for stored energy for radiating structures was given. Other approaches for stored energy are given in [15-19]. The operator for stored energy is given in [18] as a bilinear form of equation (2) as:

$$
W_{\text {sto }}=\frac{1}{4 \omega} I^{H} X^{\prime} I
$$

Where $X^{\prime}$ is given in (3) as:

$$
X^{\prime}=\omega \frac{\partial X}{\partial \omega}
$$

Thus, separating the stored energy into electric and magnetic energies in equation (4) and (5) as:

$$
\begin{gathered}
W_{m}=\frac{1}{8 \omega} I^{H} X_{m} I \\
W_{e}=\frac{1}{8 \omega} I^{H} X_{e} I
\end{gathered}
$$

Where the reactive impedance for the magnetic and electric energy is given in equation (6) and (7) respectively as:

$$
X_{m}=X^{\prime}+X
$$

and

$$
X_{e}=X^{\prime}-X
$$

Moreover, assuming that the solution for an inductive impedance matrix is decoupled from the capacitive impedance, $X$ can be expressed as :

- For inductive impedance:

$$
X=\omega L \rightarrow \frac{\partial X}{\partial \omega}=L \rightarrow X^{\prime}=\omega \frac{\partial X}{\partial \omega}=\omega L \rightarrow X^{\prime}=X
$$

- For capacitive impedance:

$$
\begin{aligned}
X=\frac{-1}{\omega C} \rightarrow \frac{\partial X}{\partial \omega}=\frac{1}{C \omega^{2}} & \rightarrow X^{\prime}=\omega \frac{\partial X}{\partial \omega}=\omega \frac{1}{C \omega^{2}} \rightarrow X^{\prime} \\
= & -X
\end{aligned}
$$

In other words, $X$ equals the absolute value of $X$ and the two possible analytical solutions depends on the nature of the stored energy whether electric or magnetic.

In a similar way, this conclusion has been demonstrated through a quantitative analysis using the first derivative of the eigenvalue with respect to the frequency [4].

\section{DISCUSSION AND CONCLUSION}

In this paper, we addressed the problem of integrating metamaterials to antennas. We have shown that the association of a metamaterial inclusion/lattice is not an straightforward task. A simplified formulation of the modal energies using the impedance matrix has been introduced. This allow any researcher to compute modal energies using any MoM commercial codes (such as FEKO [13]). Further studies of MTM inspired structures such as S-shaped inclusion and meander line will be presented during the talk.

\section{REFERENCES}

[1] A. Erentok and R.W. Ziolkowski, "Metamaterial-Inspired Efficient Electrically Small Antennas", IEEE Transactions on Antennas and Propagation, vol. 56, no. 3, pp. 691-707, 2008.

[2] Ozuem Chukwuka Seetharamdoo Divitha and Rabah M.Hassanein, "Stored energy evaluation of metamaterials based on theory of characteristic mode", IEEE-2018 12th European Conference on Antennas and Propagation (EuCAP).

[3] M. Hassanein Rabah and Divitha Seetharamdoo, "Analysis and design of metamaterial structures using the theory of characteristic modes", March 2017, pp. 2676-2680, IEEE.

[4] M. Hassanein Rabah, Divitha Seetharamdoo, and Marion Berbineau, "Analysis of Miniature Metamaterial and Magnetodielectric ArbitraryShaped Patch Antennas Using Characteristic Modes: Evaluation of the \$Q\$ Factor", IEEE Transactions on Antennas and Propagation, vol. 64, no. 7, pp. 2719-2731, July 2016.

[5] M.H. Rabah, D. Seetharamdoo, A. de Lustrac, and M. Berbineau, "Analysis of metamaterial inclusions for association with radiating elements using the theory of characteristic modes", in 2014 8th European Conference on Antennas and Propagation (EuCAP), pp. 927-930.

[6] M. H. Rabah, D. Seetharamdoo, M. Berbineau, and A. De Lustrac, "New metrics for artificial magnetism from metal-dielectric metamaterial based on the theory of characteristic modes", IEEE Antennas and Wireless Propagation Letters, vol. 15, pp. 460-463, 2016.

[7] M. H. Rabah, D. Seetharamdoo, H. Srour, and M. Berbineau, "Antenne très large bande à efficacité améliorée par l'utilisation de métamatériau: procédé de conception et prototype", French patent numéro FR3052920, 2016.

[8] R. Harrington and J. Mautz, "Theory of characteristic modes for conducting bodies", IEEE Transactions on Antennas and Propagation, vol. 19 , no. 5, pp. 622 - 628, September 1971

[9] Roger F. Harrington, J.R. Mautz, and Yu Chang, "Characteristic modes for dielectric and magnetic bodies", IEEE Transactions on Antennas and Propagation, vol. 20, no. 2, pp. 194-198, 1972.

[10] Wiley: Characteristic Modes: Theory and Applications in Antenna Engineering - Yikai Chen, Chao-Fu Wang.

[11] Miloslav Capek, Lukas Jelinek, Guy A. E. Vandenbosch, and Pavel Hazdra, "A Scheme for Stored Energy Evaluation and a Comparison with Contemporary Techniques", arXiv:1403.0572 [physics], March 2014.

[12] M. Capek, L. Jelinek, and P. Hazdra, "On the Functional Relation Between Quality Factor and Fractional Bandwidth", IEEE Transactions on Antennas and Propagation, vol. 63, no. 6, pp. 2787-2790, June 2015.

[13] FEKO, www.feko.info. 